\title{
Discrepancies Regarding the Quality of Life of Patients with Alzheimer's Disease: A Three-Year Longitudinal Study
}

\author{
Josep L. Conde-Sala ${ }^{\mathrm{a}, *}$, Oriol Turró-Garriga ${ }^{\mathrm{b}, \mathrm{c}}$, Josep Garre-Olmo ${ }^{\mathrm{b}, \mathrm{d}}$, Joan Vilalta-Franch ${ }^{\mathrm{b}, \mathrm{e}}$ \\ and Secundino Lopez-Pousa ${ }^{\text {b,e }}$ \\ ${ }^{a}$ Department of Developmental Psychology, University of Barcelona, Barcelona, Spain \\ ${ }^{\mathrm{b}}$ Research Unit, Institut d'Assistència Sanitária, Salt, Spain \\ ${ }^{\mathrm{c}}$ Department of Psychiatry and Forensic Medicine, Autonomous University of Barcelona, Cerdanyola del Vallès, \\ Spain \\ ${ }^{\mathrm{d}}$ Department of Psychology, University of Girona, Spain \\ ${ }^{\mathrm{e}}$ Memory and Dementia Assessment Unit, Institut d'Assistència Sanitaria, Salt, Spain
}

Handling Associate Editor: Pablo Martinez-Martin

Accepted 25 September 2013

\begin{abstract}
Cross-sectional studies report notable discrepancies between patient and caregiver ratings of the quality of life of patients (QoL-p) with Alzheimer's disease (AD). This study aimed to identify the factors associated with any changes in QoL-p ratings and any discrepancies between patient and caregiver ratings of QoL-p. Three-year follow-up of a cohort of noninstitutionalized patients $(n=119)$. QoL-p was assessed by the Quality of Life in AD (QoL-AD) scale. We analyzed the influence of functional and cognitive status and behavioral problems in patients, and burden and mental health in caregivers. Repeated measures analysis was applied to the scores of patients and caregivers on the QoL-AD, and to the discrepancies between them. Generally, patients' own ratings remained stable over time $\left(F_{3,116}=0.9, p=0.439\right)$, whereas caregiver ratings showed a decline $\left(F_{3,116}=9.4, p<0.001\right)$. In the analysis of discrepancies, patients with anosognosia gave higher ratings $\left(F_{1,117}=11.9, p=0.001\right)$, whereas caregiver ratings were lower when the patient showed greater agitation $\left(F_{1,117}=13.0, p<0.001\right)$, apathy $\left(F_{1,117}=15.4\right.$, $p<0.001)$, and disabilities $\left(F_{1,117}=17.1, p<0.001\right)$, and when the caregiver experienced greater burden $\left(F_{1,117}=9.0, p=0.003\right)$ and worse mental health $\left(F_{1,117}=10.1, p=0.003\right)$. Patient ratings of QoL-p remain generally stable over time, whereas those of caregivers show a decline, there being significant discrepancies in relation to specific patient and caregiver factors.
\end{abstract}

Keywords: Alzheimer's disease, anosognosia, caregiver burden, caregivers, disabilities, longitudinal study, mental health, neuropsychiatric symptoms, patients, quality of life

\section{INTRODUCTION}

Quality of life is an important concern for people with Alzheimer's disease (AD). Although their caregivers may share these concerns, they are also con-

\footnotetext{
*Correspondence to: Josep L. Conde-Sala, Department of Developmental Psychology, University of Barcelona, Passeig Vall d'Hebron, 171, 08035 Barcelona, Spain. Tel.: +34 93 3125814; Fax: +3493 4021368; E-mail: jllconde@ub.edu.
}

cerned with other more therapeutic issues, such as the medication and treatment that are available [1]. Evaluating the ability of therapeutic interventions to improve the quality of life of patients (QoL-p) with AD is now recognized as an important issue [2-5].

However, certain aspects of the factors that influence perceived QoL-p in patients and caregivers require further clarification, especially as regards the role of depression, anosognosia, and disease severity in 
patients, and mental health and burden in caregivers. Cross-sectional studies have found notable discrepancies between patient and caregiver ratings of QoL-p, and these discrepancies have been shown to increase as the dementia becomes more severe, as well as in the presence of anosognosia [6]. This raises doubts about the validity of ratings made in relation to patients with advanced dementia [7]. Burden and depression in the caregiver may also affect their ratings of QoL-p $[8,9]$. Given that we are dealing with what are essentially subjective perceptions, it is important to understand which factors may be most closely associated with differences in patient and caregiver ratings, especially since quality of life is one of the aspects considered when evaluating the suitability and effectiveness of clinical interventions. In this regard, it is also important to know whether caregiver reports offer a valid account of the patient's subjective experience.

Few longitudinal studies have yet to be carried out in this area, although they could help to clarify these discrepancies and the factors associated with them. Research involving a follow-up of more than one year has produced a consistent finding in relation to one general aspect: patient ratings of QoL-p do not change substantially during follow-up [8, 10-12], whereas caregiver ratings become significantly worse $[10,11$, $13,14]$. However, although the patient's own view does not change substantially during follow-up some studies have found that functional deficits and global impairment are related to poorer patient ratings of QoL-p, while better cognitive functioning is associated with higher ratings [12]. In another study, depression and anxiety, but not the level of cognitive functioning, were associated with a decline in ratings [8].

In terms of caregiver perceptions of QoL-p, their ratings have been found to decline as a function of certain patient factors: functional loss in activities of daily living $[11,14]$, increased neuropsychiatric symptoms $[10,11]$, a lower level of cognitive functioning [10, 14], greater depression [14], and when the caregiver's baseline rating was already lower [13].

However, studies with more than one year of follow-up have yet to examine the changes in and discrepancies between the ratings of both patients and caregivers. The present longitudinal study involved a three-year follow-up and analyzed, in both patients and caregivers, the changes in QoL-p ratings, the factors associated with these ratings, and any discrepancies between the two groups. The specific aims of the study were as follows: 1) to determine changes over time in patient and caregiver ratings of QoL-p; 2) to identify the factors associated with any changes in QoL-p ratings; and 3) to identify any discrepancies between patient and caregiver ratings of QoL-p.

\section{MATERIAL AND METHODS}

\section{Design and study population}

This was a prospective cohort study of noninstitutionalized patients with $\mathrm{AD}$ and their family caregivers over a period of three years.

All the patients were diagnosed with either AD according to DSM-IV (Diagnostic and Statistical Manual of Mental Disorders) criteria [15] or probable $\mathrm{AD}$ according to NINCDS-ADRDA (National Institute of Neurological and Communicative Disorders and Stroke/Alzheimer's Disease and Related Disorders Associations) [16]. The sample also comprised their respective family caregivers, defined as the person with main responsibility for helping the patient with activities of daily living (ADLs). Patients were excluded if they presented vascular or traumatic events, alcohol or substance dependency or abuse, and if they had severe communication problems that prevented them from responding adequately to the assessment questions.

All the AD subjects were seen as out-patients in the Memory and Dementia Assessment Unit of the Santa Caterina Hospital in Girona (Spain) and formed part of the SIDEA research project (Seguimiento Integral de la Enfermedad de Alzheimer-Comprehensive FollowUp of Alzheimer's Disease). This project is an observational, longitudinal, and pragmatic cohort study. Using a standardized protocol, it aims to describe and followup a large cohort of patients diagnosed with $\mathrm{AD}$, as well as their family caregivers, who receive outpatient medical and social care at secondary-level centers in our area. As part of this project the present study was approved by the Ethics Committee for Clinical Research of the local health authority.

The data corresponding to the baseline assessment of this study have been previously published [17].

\section{Sample power}

The sample comprised 119 patients and their respective family caregivers. This sample size was sufficient to detect (with a power of $88.9 \%$ and $99.9 \%$ in the baseline and final assessments, respectively, and accepting an alpha risk of 0.05 and a beta risk of 0.20 in a two-tailed independent contrast) a difference between patients and caregivers of 2.8 points in the baseline QoL-AD score (standard deviation $[\mathrm{SD}]=4.8$ ) and 4.6 points in the final QoL-AD score $(\mathrm{SD}=6.0)$. 


\section{Instruments}

\section{Measuring quality of life}

The Quality of Life in Alzheimer's Disease (QoLAD) scale was administered to patients and caregivers in order to assess their perception of QoL-p. The scale consists of thirteen items that reflect the subject's perception of different aspects related to wellbeing: physical health, energy, mood, living situation, memory, family, marriage, friends, self as a whole, ability to do chores around the house, ability to do things for fun, money, and life as a whole. Possible scores range from 13-52 [18], with higher scores indicating better perceived QoL.

\section{Socio-demographic data}

The socio-demographic characteristics of the patient and caregiver (age, gender, marital status, level of education, place of residence, family relationship, and whether they lived together) were recorded by means of a structured interview, the Cambridge Mental Disorders of the Elderly Examination (CAMDEX) [19].

\section{Clinical data of patients}

The clinical examination of the patient was conducted using the following instruments:

Cambridge Mental Disorders of the Elderly Examination (CAMDEX). The items used were those corresponding to the intensity of depressive symptoms (absent, mild, and moderate), the severity of dementia (mild and moderate), and anosognosia (present or absent). Data for these three variables correspond to the clinical observation of the examiner. The cognitive part of the CAMDEX, the Cambridge Cognitive Examination (CAMCOG) [19], was also applied. Possible scores range from $0-107$, with higher scores indicating better cognitive function.

Mini Mental State Examination (MMSE). This is a brief cognitive examination whose scores range from 0 to 30 [20]; the higher the score, the better the cognitive function.

Disability Assessment for Dementia (DAD). This scale was administered to caregivers and assesses a wide range of daily living activities (ADL): basic, instrumental, and leisure. It comprises 40 items and scores range from $0-80$ [21]. Higher scores are indicative of better functional status.
Neuropsychiatric Inventory (NPI). This instrument for evaluating psychiatric symptomatology in $\mathrm{AD}$ is administered to the caregiver. It comprises twelve subscales and score ranges from 0-144 [22]. The higher the score, the more symptoms are presented by the patient.

\section{Clinical data of caregivers}

The clinical examination of the caregiver was conducted using the following instruments:

SF-12 Health Survey (SF-12v1). Short form of the SF-36 Health Survey. Two global dimensions can be obtained from the direct scores: physical health and mental health, and in both dimensions the score ranges from 0-100 [23]. The higher the score, the better the respondent's health.

Zarit Burden Interview (ZBI). This questionnaire was designed to assess the burden experienced by caregivers and it comprises 22 items that are scored on a Likert scale ranging from 1 (never) to 5 (almost always). The total score therefore ranges between 22 and 110, and higher scores indicate greater burden [24].

\section{Procedure}

In the initial interview, the aims of the study were explained to patients and caregivers, who were then interviewed separately. All the patients and their caregivers gave their written consent to participate in the study. The assessment instruments were administered by a clinical neuropsychology research team from the hospital.

The baseline assessment interviews were conducted between July 2003 and July 2009. Participants in the present study were followed up at 12, 24, and 36 months after baseline.

\section{Statistical analysis}

A descriptive analysis was carried out of the clinical and socio-demographic characteristics of the sample, using absolute and relative frequencies for qualitative variables and measures of central trend and dispersion for quantitative variables. The influence of clinical and socio-demographic variables on the QoL-AD scores of patients and caregivers, and any discrepancy between these scores, was analyzed by means of parametric tests (analysis of variance $[F]$ and the Student's $t$ test $[t]$ or non-parametric tests (Mann-Whitney $\mathrm{U}[z]$ and 
Spearman $\left[r_{s}\right]$ correlations), in accordance with criteria of normality. Pearson's $\chi^{2}$ test was used for categorical variables. When there was a significant difference between two measures, Cohen's $(d)$ was calculated to determine the effect size.

In a first step we compared the clinical and sociodemographic data of the sample analyzed with the corresponding data for cases lost to follow-up. A repeated measures analysis of variance (within subjects) was then used to determine any changes over time in perceived QoL-p and for each of the patient and caregiver factors. When the sphericity assumption was not fulfilled (Mauchly's test), a multivariate approach was used. The repeated measures analyses included 119 patients and their respective caregivers, who were assessed at four time points.

In order to determine the factors associated with variation in QoL-p ratings, several regression analyses were performed, taking as the dependent variables the scores on the QoL-AD at baseline and at 36 months, as well as the differences between these two scores. These regression analyses used the Enter method (introducing all the variables in a single step). In the multiple linear regression analysis, the coefficient of contribution for each variable was calculated by means of the solution suggested by Guilford and Fruchter [25]: beta coefficient $\times$ the coefficient of correlation with the dependent variable.

The degree of agreement between patient and caregiver responses to the 13 QoL-AD items was determined by calculating weighted and unweighted kappa (k) indices; the weights used were 1.0/0.7/0.3/0.0, depending on the degree of agreement between the four categories of each item.

The factors associated with discrepancies between patient and caregiver ratings of QoL-p were identified by means of repeated measures (between-subjects) ANOVA, the dependent variable being discrepancies between patient and caregiver ratings of QoL-p at the four assessment points. The between-subjects variables were the dichotomized clinical and sociodemographic factors.

The values for partial eta-squared $\left(\eta^{2} p\right)$ were interpreted as indicating small effects $(0.01-0.05)$, medium effects (0.058-0.13), or large effects $(\geq 0.14)$ (Cohen, 1973 [26]). The $\eta_{p}^{2}$ statistic indicates the percentage of variability in the dependent variable that is explained by a given factor (range $0-1$ ). Effect sizes for differences between means (d) were defined as small (0.2-0.4), medium (0.5-0.8), or large (>0.8) [27].

For hypothesis contrasts, the level of statistical significance was set at 0.05 . All data processing and analysis was performed using SPSS 17.0 for Windows (SPSS Inc.; Chicago).

\section{RESULTS}

\section{Description of the sample}

The initial cohort included 337 participants, but of these, 218 cases were lost to follow-up, primarily due to deterioration in the patient $(43 \%)$, exitus $(15 \%)$, change of address (7\%), or for unknown reasons (35\%). The study sample therefore comprised 119 patients and their respective caregivers, all of whom completed the assessment at baseline and at 12, 24, and 36 months. The comparison of study participants with those lost to follow-up is shown in Table 1. At baseline, patients lost to follow-up were older, had greater cognitive impairment, lower functional status, more neuropsychiatric symptoms, and greater overall impairment. There were no significant differences between the two sets of caregivers.

\section{Clinical data of participants}

The global data for patients revealed a progressive deterioration over the three years of follow-up, especially as regards their cognitive functioning (MMSE and CAMCOG) and functional status (DAD). The results from the CAMDEX showed an increase in the severity of dementia, as well as in anosognosia. With respect to neuropsychiatric symptoms, there were no significant differences in the global score, but three of the subscales did show an increase over time: NPI-Anxiety $\left(F_{3,116}=4.3, p=0.006 ; \eta^{2} p=0.10\right)$, NPIApathy $\quad\left(F_{3,116}=3.9, \quad p=0.010 ; \quad \eta_{p}^{2}=0.09\right)$, and NPI-Aberrant motor behavior $\left(F_{3,116}=3.0, p=0.030\right.$; $\left.\eta^{2}{ }_{p}=0.07\right)$. Among caregivers, the only variable that showed a progressive and significant increase over time was burden (ZBI) (see Table 2).

Patients' own ratings of QoL-p did not differ significantly across the four assessment points $\left(F_{3,116}=0.9\right.$, $\left.p=0.439 ; \eta^{2}{ }_{p}=0.02\right)$. By contrast, caregiver ratings showed a general decline $\left(F_{3,116}=9.4, p<0.001\right.$; $\left.\eta_{p}^{2}=0.19\right)$. The overall discrepancy between patient and caregiver ratings increased over the 36 months of follow-up $\left(F_{3,116}=6.4, p<0.001 ; \eta^{2}{ }_{p}=0.14\right)$, and especially after 12 months, coinciding with more severe dementia (baseline differences: $t_{118}=6.3, p<$ $0.001, \eta^{2} p=0.25$; at 12 months: $t_{118}=5.4, p<0.001$, $\eta_{p}^{2}=0.20$; at 24 months: $t_{118}=6.6, p<0.001, \eta^{2}=$ 0.27 ; at 36 months: $t_{118}=8.3, p<0.001, \eta_{p}^{2}=0.36$ ). 
Table 1

Comparison (at baseline) of the two groups

\begin{tabular}{|c|c|c|c|c|c|}
\hline & \multirow{2}{*}{$\begin{array}{l}\text { Study participants } \\
\qquad(n=119)\end{array}$} & \multirow{2}{*}{$\begin{array}{l}\text { Lost to follow-up } \\
\qquad(n=218)\end{array}$} & \multicolumn{3}{|c|}{ Differences } \\
\hline & & & Test (df) & $p$ & $d$ \\
\hline \multicolumn{6}{|l|}{ Patient factors } \\
\hline Age (years) & $77.0 \pm 6.7$ & $78.6 \pm 6.8$ & $2.46^{\mathrm{a}}$ & 0.014 & 0.23 \\
\hline Women $(\%)$ & $76(63.9)$ & $144(66.1)$ & $0.16(1)^{\mathrm{b}}$ & 0.687 & \\
\hline Education, $\geq 6$ years $(\%)$ & $72(60.5)$ & $124(56.9)$ & $0.41(1)^{\mathrm{b}}$ & 0.519 & \\
\hline MMSE (cognition) & $18.9 \pm 3.6$ & $17.3 \pm 4.5$ & $3.00^{\mathrm{a}}$ & 0.003 & 0.39 \\
\hline CAMCOG (cognition) & $58.9 \pm 9.9$ & $52.9 \pm 12.7$ & $4.31^{\mathrm{a}}$ & $<0.001$ & 0.52 \\
\hline $\mathrm{DAD}(\mathrm{ADL})$ & $68.7 \pm 7.8$ & $65.5 \pm 8.6$ & $3.34^{\mathrm{a}}$ & 0.001 & 0.38 \\
\hline NPI (behavioral) & $9.4 \pm 9.4$ & $12.4 \pm 13.3$ & $1.99^{\mathrm{a}}$ & 0.047 & 0.26 \\
\hline \multicolumn{6}{|l|}{ CAMDEX $(\%)$} \\
\hline Depression, mild-moderate & $17(14.3)$ & $40(18.3)$ & $1.39(2)^{\mathrm{b}}$ & 0.498 & \\
\hline Dementia, moderate & $12(10.1)$ & $42(19.3)$ & $7.78(2)^{\mathrm{b}}$ & 0.020 & \\
\hline Anosognosia & $11(9.2)$ & $22(10.1)$ & $0.06(1)^{\mathrm{b}}$ & 0.802 & \\
\hline \multicolumn{6}{|l|}{ Caregiver factors } \\
\hline Age (years) & $61.0 \pm 14.6$ & $58.8 \pm 14.2$ & $1.42^{\mathrm{a}}$ & 0.155 & \\
\hline Women $(\%)$ & $82(68.9)$ & $150(68.8)$ & $0.00(1)^{\mathrm{b}}$ & 0.985 & \\
\hline Education, $\geq 6$ years $(\%)$ & $94(79.0)$ & $175(80.3)$ & $0.07(1)^{\mathrm{b}}$ & 0.779 & \\
\hline ZBI (burden) & $39.7 \pm 12.5$ & $41.9 \pm 12.8$ & $1.74^{\mathrm{a}}$ & 0.082 & \\
\hline SF-12 (physical health) & $49.9 \pm 9.2$ & $50.2 \pm 8.9$ & $0.14^{\mathrm{a}}$ & 0.888 & \\
\hline SF-12 (mental health) & $46.7 \pm 11.0$ & $45.7 \pm 11.2$ & $0.78^{\mathrm{a}}$ & 0.434 & \\
\hline
\end{tabular}

${ }^{a}$ Mann-Whitney $U$ test; ${ }^{b}$ Pearson $\chi^{2}$ test; $d$, Cohen's d. $p$-values $<0.05$ are shown in bold. MMSE, Mini Mental State Examination; CAMCOG, Cambridge Cognitive Examination; CAMDEX, Cambridge Mental Disorders of the Elderly Examination; ADL, Activities of daily living; DAD, Disability Assessment for Dementia; NPI, Neuropsychiatric Inventory; ZBI, Zarit Burden Inventory; SF-12, Short Form of Health Survey.

Table 2

Clinical data of participants

\begin{tabular}{|c|c|c|c|c|c|c|c|}
\hline & \multirow{2}{*}{$\begin{array}{c}\text { Baseline (1) } \\
(n=119)\end{array}$} & \multirow{2}{*}{$\begin{array}{c}12 \text { months (2) } \\
(n=119)\end{array}$} & \multirow{2}{*}{$\begin{array}{c}24 \text { months }(3) \\
(n=119)\end{array}$} & \multirow{2}{*}{$\begin{array}{c}36 \text { months (4) } \\
(n=119)\end{array}$} & \multicolumn{3}{|c|}{ Within-subjects } \\
\hline & & & & & $F$ & $p$ & $\eta_{p}^{2}$ \\
\hline \multicolumn{8}{|l|}{$\overline{\text { QoL-AD }(\text { mean } \pm \mathrm{SD})}$} \\
\hline Patient & $34.7 \pm 4.6$ & $35.2 \pm 4.5$ & $34.8 \pm 4.8$ & $35.1 \pm 5.0$ & 0.9 & 0.439 & 0.02 \\
\hline Caregiver & $31.9 \pm 5.0$ & $32.7 \pm 5.0$ & $31.1 \pm 5.3^{\mathrm{d}}$ & $30.5 \pm 4.9^{\mathrm{c}, \mathrm{e}}$ & 9.4 & $<0.001$ & 0.19 \\
\hline Discrepancy QoL & $2.8 \pm 4.8$ & $2.4 \pm 4.9$ & $3.6 \pm 6.0$ & $4.6 \pm 6.0^{\mathrm{c}, \mathrm{e}}$ & 6.4 & $<0.001$ & 0.14 \\
\hline \multicolumn{8}{|l|}{ Patient factors (mean $\pm \mathrm{SD}$ ) } \\
\hline MMSE (cognition) & $18.9 \pm 3.6$ & $18.5 \pm 3.4$ & $18.3 \pm 4.7$ & $15.3 \pm 5.2^{\mathrm{c}, \mathrm{e}, \mathrm{f}}$ & 37.9 & $<0.001$ & 0.49 \\
\hline CAMCOG (cognition) & $58.9 \pm 9.9$ & $56.5 \pm 11.2^{\mathrm{a}}$ & $53.4 \pm 12.9^{\mathrm{b}, \mathrm{d}}$ & $48.6 \pm 16.7^{\mathrm{c}, \mathrm{e}, \mathrm{f}}$ & 24.5 & $<0.001$ & 0.38 \\
\hline DAD (function) & $69.8 \pm 7.7$ & $66.9 \pm 9.5^{\mathrm{a}}$ & $63.3 \pm 9.9^{\mathrm{b}, \mathrm{d}}$ & $59.1 \pm 10.5^{\mathrm{c}, \mathrm{e}, \mathrm{f}}$ & 52.5 & $<0.001$ & 0.57 \\
\hline NPI (behavior) & $9.4 \pm 9.4$ & $9.0 \pm 12.3$ & $10.4 \pm 11.7$ & $11.1 \pm 11.2$ & 1.1 & 0.341 & 0.02 \\
\hline Agitation & $0.8 \pm 1.8$ & $0.6 \pm 1.9$ & $1.0 \pm 2.5$ & $1.2 \pm 2.9$ & 1.3 & 0.266 & 0.03 \\
\hline Depression & $1.5 \pm 2.8$ & $1.0 \pm 2.5$ & $0.9 \pm 2.2$ & $0.7 \pm 1.9^{c}$ & 2.6 & 0.051 & 0.06 \\
\hline Anxiety & $0.7 \pm 1.7$ & $0.8 \pm 2.2$ & $0.5 \pm 1.8$ & $1.4 \pm 2.8^{\mathrm{f}}$ & 4.3 & 0.006 & 0.10 \\
\hline Apathy & $2.2 \pm 3.0$ & $2.1 \pm 3.3$ & $2.9 \pm 4.0$ & $3.1 \pm 3.9^{\mathrm{e}}$ & 3.9 & 0.010 & 0.09 \\
\hline Aberrant motor & $0.2 \pm 1.1$ & $0.3 \pm 1.8$ & $0.8 \pm 2.4$ & $0.7 \pm 2.3$ & 3.0 & 0.030 & 0.07 \\
\hline \multicolumn{8}{|l|}{$\operatorname{CAMDEX}(n, \%)$} \\
\hline Depression, mild-mod. & $17(14.3)$ & $12(10.1)$ & $16(13.4)$ & $20(16.8)$ & 2.0 & 0.569 & \\
\hline Dementia, moderate & $13(10.9)$ & $29(24.4)$ & $45(37.8)$ & $66(55.5)$ & 40.2 & $<0.001$ & \\
\hline Anosognosia & $11(9.2)$ & $16(13.4)$ & $23(19.3)$ & $32(26.9)$ & 12.1 & 0.006 & \\
\hline \multicolumn{8}{|c|}{ Caregiver factors (mean \pm SD) } \\
\hline ZBI (burden) & $39.7 \pm 12.4$ & $40.3 \pm 11.5$ & $42.7 \pm 13.9^{\mathrm{b}, \mathrm{d}}$ & $43.5 \pm 13.6^{\mathrm{c}, \mathrm{e}}$ & 5.6 & 0.001 & 0.12 \\
\hline SF-12 (physical health) & $49.9 \pm 9.2$ & $49.6 \pm 9.1$ & $49.2 \pm 9.4)$ & $49.1 \pm 9.3$ & 0.3 & 0.771 & 0.01 \\
\hline SF-12 (mental health) & $46.7 \pm 11.0$ & $45.9 \pm 11.0$ & $45.2 \pm 10.8$ & $45.7 \pm 11.3$ & 0.5 & 0.656 & 0.01 \\
\hline
\end{tabular}

$F$, ANOVA, repeated measures $(\mathrm{df}=3,116) ; \eta^{2}$, partial eta squared; Pearson $\chi^{2}$ test $(\mathrm{df}=3)$ for categorical variables. Significant with Bonferroni post-hoc, Contrasts $={ }^{\mathrm{a}} 1-2,{ }^{\mathrm{b}} 1-3,{ }^{\mathrm{c}} 1-4,{ }^{\mathrm{d}} 2-3,{ }^{\mathrm{e}} 2-4,{ }^{\mathrm{f}} 3-4$. $p$-values $<0.05$ are shown in bold. QoL-AD, Quality of Life-Alzheimer's Disease scale; MMSE, Mini Mental State Examination; CAMCOG, Cambridge Cognitive Examination; CAMDEX, Cambridge Mental Disorders of the Elderly Examination; DAD, Disability Assessment for Dementia; NPI, Neuropsychiatric Inventory; ZBI, Zarit Burden Inventory; SF-12, Short Form of Health Survey. 
Table 3

Socio-demographic data and quality of life of patients

\begin{tabular}{|c|c|c|c|c|c|c|c|c|c|}
\hline & \multirow[t]{2}{*}{$n$} & \multirow{2}{*}{$\begin{array}{c}\text { Baseline (1) } \\
(n=119)\end{array}$} & \multirow{2}{*}{$\begin{array}{c}12 \text { months (2) } \\
(n=119)\end{array}$} & \multirow{2}{*}{$\begin{array}{c}24 \text { months }(3) \\
(n=119)\end{array}$} & \multirow{2}{*}{$\begin{array}{c}36 \text { months (4) } \\
(n=119)\end{array}$} & \multicolumn{3}{|c|}{ Differences } & \multirow[b]{2}{*}{$\eta_{p}^{2}$} \\
\hline & & & & & & $F$ & & $p$ & \\
\hline \multicolumn{10}{|c|}{ QoL-AD patient } \\
\hline \multicolumn{10}{|l|}{ Gender } \\
\hline Men & 43 & $36.1 \pm 4.8$ & $36.5 \pm 4.3$ & $35.9 \pm 5.7$ & $35.8 \pm 5.3$ & W & 0.5 & 0.627 & 0.01 \\
\hline Women & 76 & $33.9 \pm 4.3$ & $34.5 \pm 4.6$ & $34.2 \pm 4.2$ & $34.7 \pm 4.8$ & W & 0.8 & 0.444 & 0.01 \\
\hline$z(p)$ & & $2.2(\mathbf{0 . 0 2 2})$ & $2.3(\mathbf{0 . 0 1 8})$ & $1.8(0.061)$ & $1.0(0.316)$ & $\mathrm{B}$ & 5.2 & 0.023 & 0.04 \\
\hline \multicolumn{10}{|l|}{ Schooling } \\
\hline$<6$ years & 47 & $35.0 \pm 4.7$ & $35.5 \pm 4.5$ & $34.8 \pm 4.5$ & $35.5 \pm 5.0$ & $\mathrm{~W}$ & 0.6 & 0.616 & 0.01 \\
\hline $6-8$ years & 56 & $34.2 \pm 4.6$ & $34.4 \pm 4.5$ & $34.3 \pm 5.1$ & $34.0 \pm 4.8$ & W & 0.2 & 0.845 & 0.00 \\
\hline$>8$ years & 16 & $35.8 \pm 4.6$ & $37.2 \pm 4.3$ & $36.8 \pm 4.7$ & $38.1 \pm 4.5$ & W & 1.3 & 0.276 & 0.08 \\
\hline$\chi^{2}(p)$ & & $2.9(0.232)$ & $4.7(0.092)$ & $4.3(0.115)$ & $9.3(\mathbf{0 . 0 0 9})$ & B & 3.1 & 0.048 & 0.05 \\
\hline \multicolumn{10}{|c|}{ QoL-AD caregivers } \\
\hline \multicolumn{10}{|l|}{ Gender } \\
\hline Men & 37 & $31.3 \pm 5.1$ & $32.0 \pm 5.1$ & $30.9 \pm 5.3$ & $30.2 \pm 6.2$ & W & 1.9 & 0.131 & 0.05 \\
\hline Women & 82 & $32.1 \pm 4.9$ & $33.1 \pm 4.9$ & $31.2 \pm 5.3^{\mathrm{d}}$ & $30.7 \pm 4.3^{\mathrm{e}}$ & W & 8.0 & $<0.001$ & 0.09 \\
\hline$t(p)$ & & $0.8(0.413)$ & $1.0(0.299)$ & $0.3(0.762)$ & $0.5(0.605)$ & B & 0.6 & 0.419 & 0.00 \\
\hline \multicolumn{10}{|l|}{ Schooling } \\
\hline$<6$ years & 24 & $31.1 \pm 4.5$ & $32.6 \pm 4.3$ & $30.6 \pm 6.3$ & $29.9 \pm 6.6$ & W & 1.6 & 0.189 & 0.06 \\
\hline $6-8$ years & 34 & $32.3 \pm 5.3$ & $32.1 \pm 5.0$ & $31.7 \pm 5.0$ & $30.2 \pm 4.6^{\mathrm{e}}$ & W & 3.5 & 0.017 & 0.09 \\
\hline$>8$ years & 61 & $32.0 \pm 5.0$ & $33.2 \pm 5.2$ & $31.1 \pm 5.1^{\mathrm{d}}$ & $30.9 \pm 4.4^{\mathrm{e}}$ & W & 6.6 & $<0.001$ & 0.10 \\
\hline$F(p)$ & & $0.4(0.658)$ & $0.5(0.585)$ & $0.3(0.716)$ & $0.4(0.627)$ & B & 0.2 & 0.764 & 0.00 \\
\hline \multicolumn{10}{|l|}{ Relationship } \\
\hline Husband & 22 & $31.8 \pm 4.8$ & $33.5 \pm 3.8$ & $32.8 \pm 4.8$ & $32.1 \pm 6.3$ & W & 1.1 & 0.338 & 0.05 \\
\hline Daughter & 40 & $32.3 \pm 4.5$ & $33.1 \pm 4.7$ & $32.0 \pm 5.0$ & $31.8 \pm 3.9$ & W & 1.5 & 0.218 & 0.03 \\
\hline Wife & 33 & $32.7 \pm 5.1$ & $32.9 \pm 5.6$ & $29.9 \pm 5.7^{d}$ & $29.4 \pm 4.5^{\mathrm{c}, \mathrm{e}}$ & W & 8.2 & $<0.001$ & 0.20 \\
\hline Son & 15 & $30.6 \pm 5.8$ & $29.9 \pm 6.2$ & $28.2 \pm 5.0$ & $27.3 \pm 5.1$ & W & 2.8 & 0.048 & 0.17 \\
\hline$F(p)$ & & $0.6(0.576)$ & $1.7(0.165)$ & $3.2(\mathbf{0 . 0 2 5})$ & $4.5(\mathbf{0 . 0 0 5})$ & $\mathrm{B}$ & 2.7 & 0.046 & 0.07 \\
\hline
\end{tabular}

$F$, ANOVA; $\eta_{p}^{2}$, partial eta squared; $z$, Mann-Whitney $\mathrm{U}$ test; $\chi^{2}$, Chi-squared test; $t$, Student's $t$ test. $p$-values $<0.05$ are shown in bold. B, Between-subjects; W, Within-subjects. Significant with Bonferroni post-hoc, Contrasts = ${ }^{\mathrm{a}} 1-2,{ }^{\mathrm{b}} 1-3,{ }^{\mathrm{c}} 1-4,{ }^{\mathrm{d}} 2-3,{ }^{\mathrm{e}} 2-4,{ }^{\mathrm{f}} 3-4$. QoL-AD, Quality of Life-Alzheimer's Disease scale.

\section{Socio-demographic data and perceived QoL}

In the repeated measures (between-subjects) analysis of patient data, the most important differences were related to two factors: gender, with men giving higher ratings than women of their own $\mathrm{QoL}$ $\left(F_{1,117}=5.2, p=0.023, \eta_{p}^{2}=0.04\right)$, and level of education, with higher ratings of QoL being given by the more educated patients $\left(F_{2,116}=3.1, p=0.048\right.$, $\left.\eta^{2}{ }_{p}=0.05\right)$. No significant differences were observed as regards the relationship to the caregiver, the patient's age, or whether or not patient and caregiver lived together.

In the repeated measures (between subjects) analysis of caregiver data, the most important difference concerned the relationship between patient and caregiver, with wives and sons giving lower ratings of QoL-p than did husbands and daughters $\left(F_{3,106}=2.7, p=0.046\right.$, $\eta^{2}{ }_{p}=0.07$ ). Although there were no overall differences related to gender, female caregivers showed a greater decline in their scores over the three-year follow-up period. The same trend was observed among caregivers with the highest level of education, whose ratings of
QoL-p also showed the greatest decline over the three years. No significant differences were observed in relation to the caregiver's age or whether or not patient and caregiver lived together. The complete data are shown in Table 3.

\section{Factors associated with changes in patient and caregiver ratings of $Q o L-p$}

The multivariate linear regression analysis identified the factors associated with perceived QoL-p at baseline and 36 months later, as well as the changes that occurred during the follow-up period (Table 4).

\section{Patients}

At baseline the factors associated with a better perceived quality of life were less depression, male gender, and less apathy. In the final assessment, male gender and, especially, less depression continued to be associated with a more positive view of QoL-p, although at 36 months the following factors also showed an association: higher functional status, greater disinhibition, and greater anosognosia. The differences between baseline 


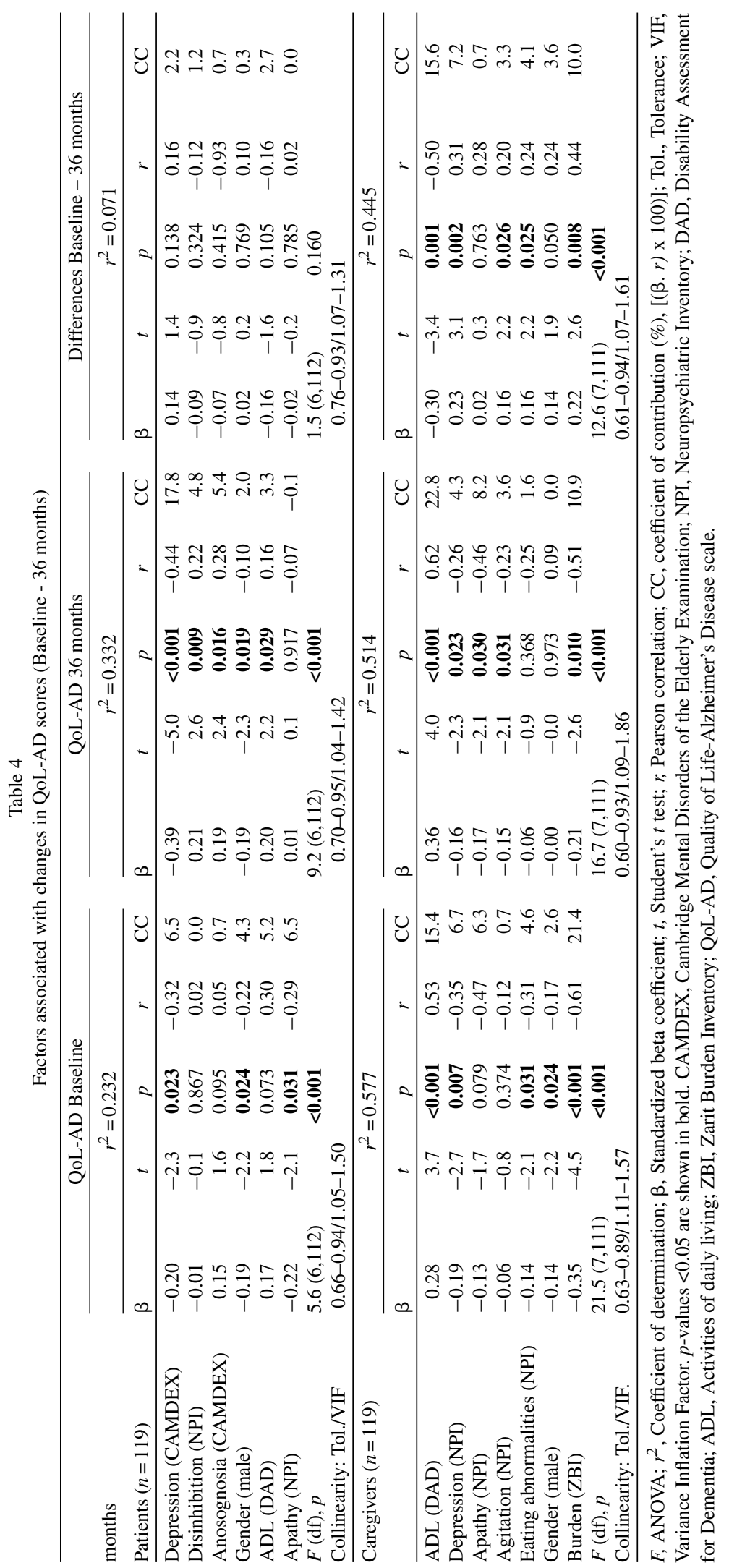




\begin{tabular}{|c|c|c|c|c|c|c|c|c|}
\hline & \multirow[t]{2}{*}{$\begin{array}{c}\text { (Cut-off) } \\
n\end{array}$} & \multirow[t]{2}{*}{$\begin{array}{c}\text { Baseline } \\
\text { Mean } \pm \text { SD }\end{array}$} & \multirow[t]{2}{*}{$\begin{array}{l}12 \text { months } \\
\text { Mean } \pm \text { SD }\end{array}$} & \multirow[t]{2}{*}{$\begin{array}{l}24 \text { months } \\
\text { Mean } \pm \text { SD }\end{array}$} & \multirow[t]{2}{*}{$\begin{array}{c}36 \text { months } \\
\text { Mean } \pm \text { SD }\end{array}$} & \multicolumn{3}{|c|}{$\begin{array}{l}\text { Patients-caregivers } \\
\text { between-subjects }\end{array}$} \\
\hline & & & & & & $F$ & $p$ & $\eta_{p}^{2}$ \\
\hline \multicolumn{9}{|l|}{ Patient factors } \\
\hline DAD. ADL & $( \pm 65)$ & & & & & 17.1 & $<0.001$ & 0.12 \\
\hline Low disability & 66 & $1.5 \pm 4.3$ & $1.0 \pm 3.9$ & $2.3 \pm 5.5$ & $3.1 \pm 5.7$ & & & \\
\hline High disability & 53 & $4.3 \pm 5.1$ & $4.3 \pm 5.5$ & $5.3 \pm 6.3$ & $6.4 \pm 6.0$ & & & \\
\hline$t(p)$ & & $3.2(\mathbf{0 . 0 0 2})$ & $3.7(<\mathbf{0 . 0 0 1})$ & 2.7 (0.008) & $3.0(\mathbf{0 . 0 0 3})$ & & & \\
\hline NPI. Apathy & $( \pm 2.6)$ & & & & & 15.4 & $<0.001$ & 0.11 \\
\hline Low apathy & 69 & $2.2 \pm 4.9$ & $1.0 \pm 4.2$ & $2.1 \pm 5.7$ & $3.2 \pm 6.1$ & & & \\
\hline High apathy & 50 & $3.6 \pm 4.7$ & $4.4 \pm 5.2$ & $5.7 \pm 5.9$ & $6.5 \pm 5.3$ & & & \\
\hline$t(p)$ & & $1.5(\mathbf{0 . 1 1 7})$ & $3.9(<\mathbf{0 . 0 0 1})$ & $3.3(\mathbf{0 . 0 0 1})$ & $3.0(\mathbf{0 . 0 0 3})$ & & & \\
\hline NPI. Agitation & $( \pm 0.9)$ & & & & & 13.0 & $<0.001$ & 0.10 \\
\hline Low agitation & 79 & $1.8 \pm 4.7$ & $1.6 \pm 4.9$ & $2.6 \pm 6.0$ & $3.4 \pm 5.7$ & & & \\
\hline High agitation & 40 & $4.6 \pm 4.7$ & $4.0 \pm 4.7$ & $5.7 \pm 5.5$ & $6.8 \pm 6.1$ & & & \\
\hline$t(p)$ & & $3.0(\mathbf{0 . 0 0 3})$ & $2.4(\mathbf{0 . 0 1 6})$ & $2.6(\mathbf{0 . 0 0 9})$ & $2.9(\mathbf{0 . 0 0 4})$ & & & \\
\hline Anosognosia & & & & & & 11.9 & 0.001 & 0.09 \\
\hline No & 74 & $1.8 \pm 4.6$ & $1.5 \pm 4.5$ & $2.4 \pm 5.7$ & $2.7 \pm 5.4$ & & & \\
\hline Yes & 45 & $4.4 \pm 4.8$ & $4.0 \pm 5.2$ & $4.8 \pm 5.8$ & $7.6 \pm 5.8$ & & & \\
\hline$t(p)$ & & $2.8(\mathbf{0 . 0 0 5})$ & $2.7(\mathbf{0 . 0 0 6})$ & $2.2(0.477)$ & $4.5(<\mathbf{0 . 0 0 1})$ & & & \\
\hline CAMCOG & $( \pm 55)$ & & & & & 0.6 & 0.427 & 0.00 \\
\hline Low decline & 62 & $3.2 \pm 4.6$ & $2.0 \pm 4.5$ & $4.0 \pm 5.7$ & $2.9 \pm 5.4$ & & & \\
\hline High decline & 57 & $2.4 \pm 5.1$ & $2.8 \pm 5.3$ & $3.3 \pm 6.3$ & $6.1 \pm 6.2$ & & & \\
\hline$t(p)$ & & $0.8(0.405)$ & $0.9(0.349)$ & $0.6(0.528)$ & $2.8(\mathbf{0 . 0 0 5})$ & & & \\
\hline \multicolumn{9}{|l|}{ Caregiver factors } \\
\hline ZBI. Burden & $( \pm 42)$ & & & & & 9.0 & 0.003 & 0.07 \\
\hline Low burden & 68 & $1.8 \pm 4.9$ & $1.6 \pm 4.7$ & $2.2 \pm 5.8$ & $3.7 \pm 6.0$ & & & \\
\hline High burden & 51 & $4.1 \pm 4.6$ & $3.5 \pm 5.1$ & $5.5 \pm 5.9$ & $5.7 \pm 5.8$ & & & \\
\hline$t(p)$ & & $2.5(\mathbf{0 . 0 1 3})$ & $2.0(\mathbf{0 . 0 4 3})$ & $2.9(\mathbf{0 . 0 0 4})$ & $1.7(0.083)$ & & & \\
\hline SF-12. Mental & $( \pm 46)$ & & & & & 10.1 & 0.002 & 0.08 \\
\hline Better health & 72 & $1.7 \pm 4.6$ & $1.7 \pm 4.4$ & $2.6 \pm 5.5$ & $3.5 \pm 5.8$ & & & \\
\hline Worse health & 47 & $4.5 \pm 4.8$ & $3.6 \pm 5.5$ & $5.2 \pm 6.5$ & $6.2 \pm 6.0$ & & & \\
\hline$t(p)$ & & 3.1 (0.002) & $2.0(\mathbf{0 . 0 4 3})$ & $2.2(\mathbf{0 . 0 2 4})$ & $2.3(\mathbf{0 . 0 1 8})$ & & & \\
\hline
\end{tabular}

Note: The values indicate the difference between the QoL-p ratings of patients and caregivers at each assessment point and in the sub-groups (high vs. low scores) for each factor. The cut-off point is the mean of the four assessments for continuous variables. $F$, Repeated measures $(\mathrm{df}=1,117) ; \eta^{2}{ }_{p}$, partial eta squared; $t$, Student's $t$ test $(\mathrm{df}=117)$. $p$-values $<0.05$ are shown in bold. CAMDEX (Anosognosia), Cambridge Mental Disorders of the Elderly Examination; CAMCOG, Cambridge Cognitive Examination; NPI, Neuropsychiatric Inventory; DAD, Disability Assessment for Dementia; ADL, Activities of daily living; ZBI, Zarit burden Inventory; SF-12, Short Form of Health Survey; QoL-AD, Quality of Life-Alzheimer's Disease scale.

and final scores on the QoL-AD were not significantly associated with any factor.

\section{Caregivers}

At baseline the main factors associated with higher ratings of QoL-p were better functional status in the patient and less burden in the caregiver; other associated patient factors were less depression, fewer appetite/eating abnormalities, and male gender. In the final assessment, better functional status in the patient and less burden in the caregiver continued to be relevant, with other associated patient factors being less agitation, depression, and apathy. The decline (baseline-final) in caregiver ratings of QoL-p was particularly related to lower functional status and greater depression in the patient, as well as to greater caregiver burden; other associated patient factors were greater agitation and appetite/eating abnormalities.
Functional impairment $\left(r_{s}\right.$, Spearman coefficient $=-0.48$ to $-0.55, p<0.001)$ and neuropsychiatric symptoms $\left(r_{s}=0.41\right.$ to $\left.0.53, p<0.001\right)$ showed a high correlation with caregiver burden, which in turn was correlated with a more negative view of QoL-p among caregivers $(r$, Pearson correlation $=-0.51$ to $-0.67, p<0.001)$ at the four assessment points.

\section{Degree of agreement between patient and caregiver scores on the QoL-AD}

The degree of agreement (kappa index) between patient and caregiver ratings of the patient's quality of life was poor for most of the 13 items: values for unweighted and weighted kappa were, respectively, $\leq 0.21$ and $\leq 0.25$. The items with the highest mean kappa value (unweighted/weighted) at the four assessment points were $\operatorname{Mood}(k=0.20 / 0.25)$ 


\section{PATIENT FACTORS}

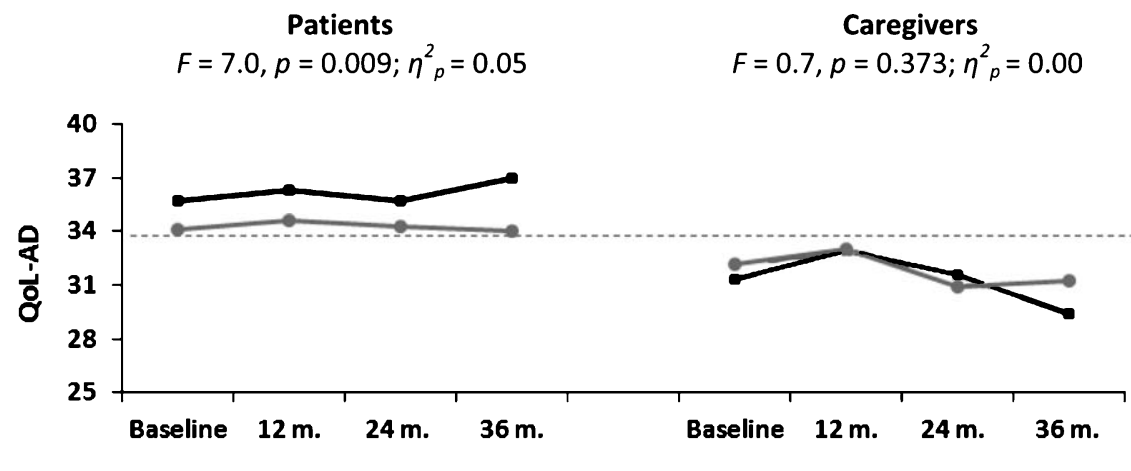

Discrepancy $F=11.9, p=0.001 ; \eta_{p}^{2}=0.09$
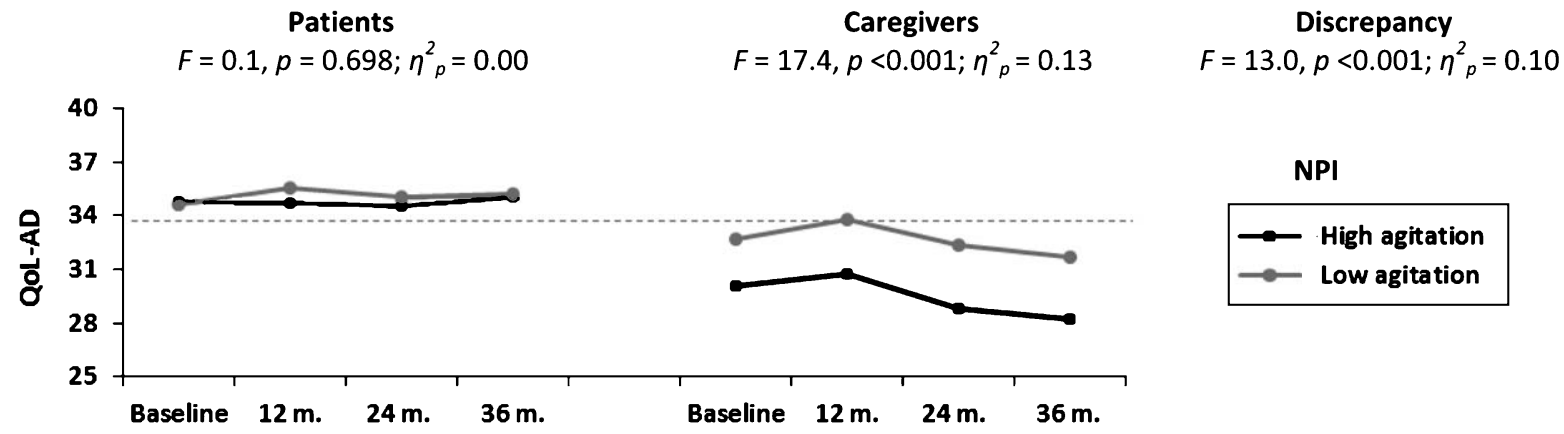

\section{CAMDEX}

Anosognosia

No anosognosia
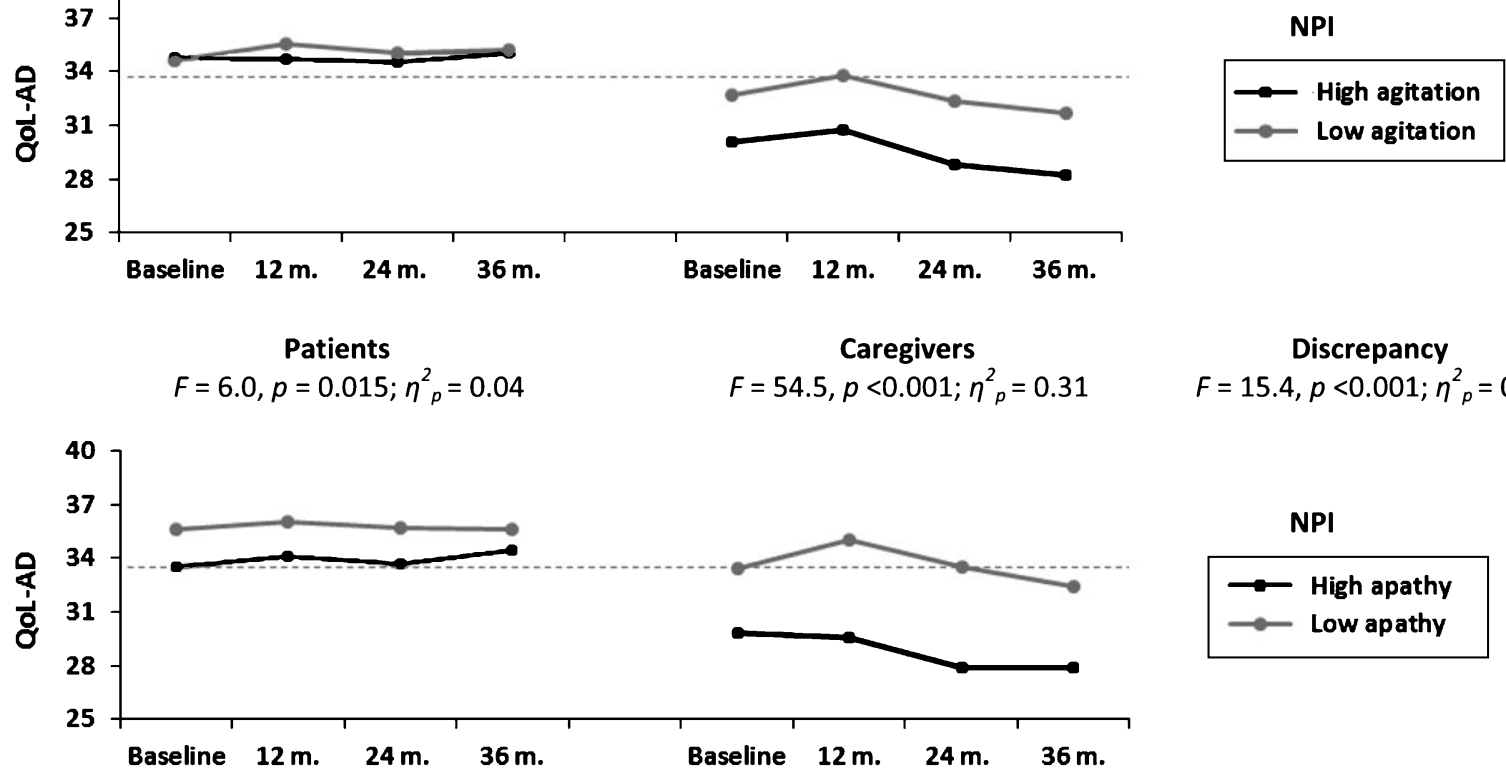

Discrepancy $F=15.4, p<0.001 ; \eta_{p}^{2}=0.11$

Patients

$F=11.8, p=0.001 ; \eta_{p}^{2}=0.09$

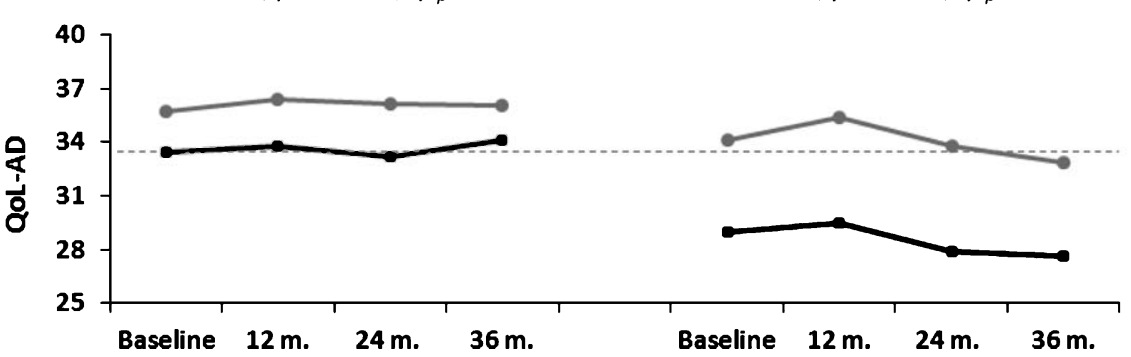

NPI

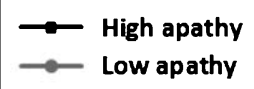

Discrepancy $F=17.1, p<0.001 ; \eta_{p}^{2}=0.12$

DAD

High disability Low disability

Fig. 1. Patient factors. Patient and caregiver scores on the QoL-AD in relation to the influence of the most relevant patient factors. F, ANOVA, repeated measures (between-subjects); df $(1,117) ; \eta_{p}^{2}$, partial eta squared; QoL-AD, Quality of Life-Alzheimer's Disease scale; CAMDEX, Cambridge Mental Disorders of the Elderly Examination; NPI, Neuropsychiatric Inventory; DAD, Disability Assessment for Dementia. 


\section{CAREGIVER FACTORS}

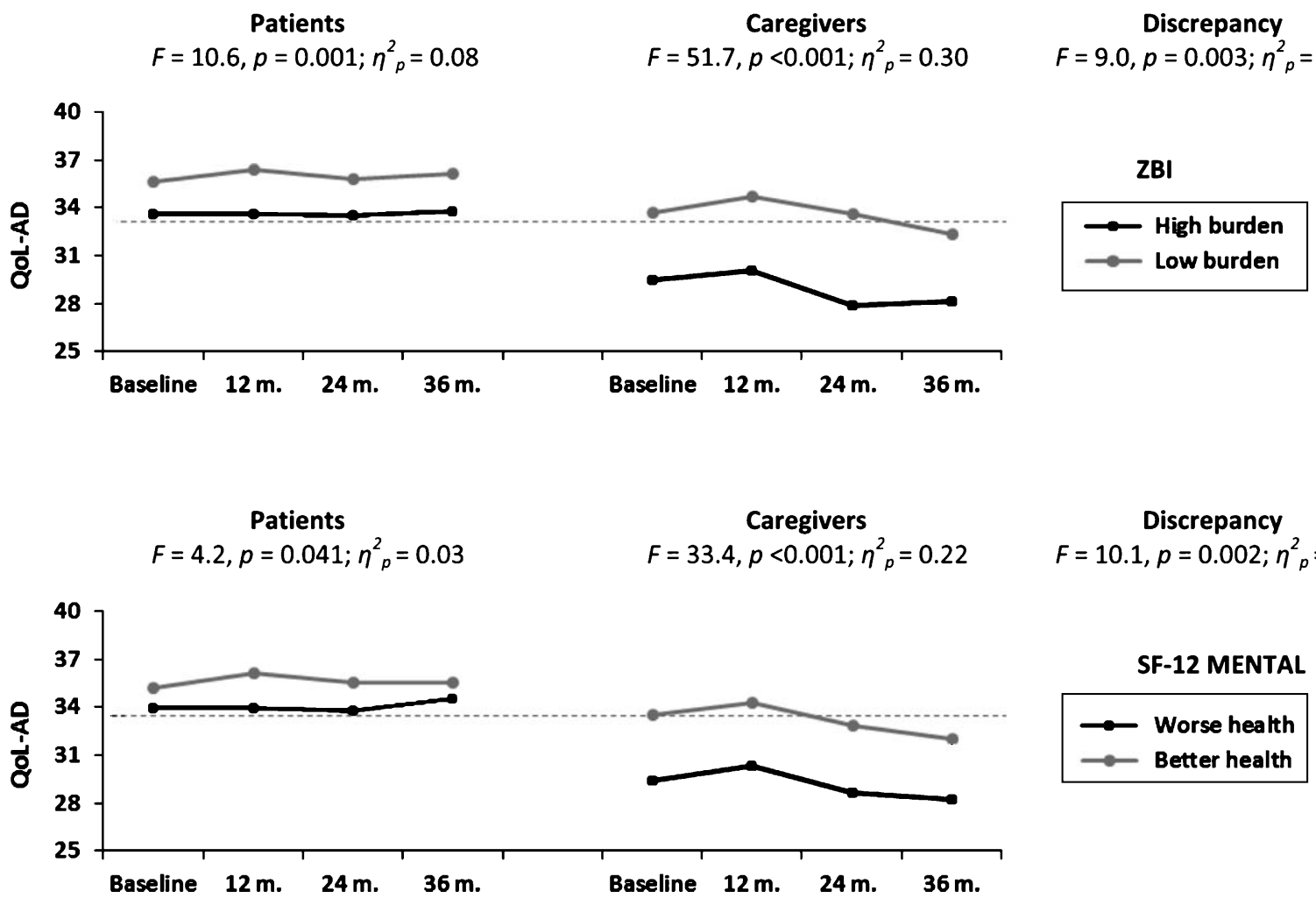

Fig. 2. Caregiver factors. Patient and caregiver scores on the QoL-AD in relation to the influence of the most relevant caregiver factors. F, ANOVA, repeated measures (between-subjects); df $(1,117) ; \eta^{2}{ }_{p}$, partial eta squared; QoL-AD, Quality of Life-Alzheimer's Disease scale; ZBI, Zarit burden Inventory; SF-12, Short Form of Health Survey.

and Marriage $(k=0.21 / 0.25)$, while the lowest means corresponded to Living situation $(k=0.07 / 0.07)$ and Memory $(k=0.09 / 0.10)$. The mean kappa index decreased progressively in magnitude across the four assessment points, this being the case for both unweighted $(k=0.17 / 0.13 / 0.11 / 0.10)$ and weighted values $(k=0.20 / 0.17 / 0.15 / 0.14)$.

\section{Discrepancies between patient and caregiver scores on the QoL-AD}

There was a general discrepancy between patients and caregivers in terms of their total score on the QoL$\operatorname{AD}\left(F_{1,236}=40.8, p<0.001 ; \eta^{2} p=0.14\right)$.

More specific discrepancies, related with the various factors, were examined by means of a repeated measures (between-subjects) analysis of variance, in which the dependent variable was the discrepancy between patient and caregiver scores on the QoL-AD at the four assessment points. The between-subjects groups were formed by dichotomizing the factors as follows: for continuous variables we took the mean score at each assessment point, while for categorical variables we used the characteristics of subjects (Table 5).

The most notable discrepancies $\left(\eta_{p}^{2}>0.06\right)$ between patient and caregiver ratings were observed in the presence of anosognosia, which was associated with higher scores in the patient, and agitation, apathy, and functional deficits, which were associated with lower ratings among caregivers. A number of caregiver factors were also significantly related to lower ratings of QoL-p, namely greater burden and poorer mental health.

Figure 1 shows patient and caregiver scores on the QoL-AD in relation to the patient factors that were shown to be relevant: anosognosia (CAMDEX), apathy and agitation (NPI), and functional capacity (DAD). Similarly, Fig. 2 shows patient and caregiver scores on the QoL-AD in relation to the caregiver factors that were shown to be relevant: burden (ZBI) and mental health (SF-12). These figures also show the statistical test values for differences among both patients and 
caregivers, as well as the significance of any discrepancies between patient and caregiver scores.

It should be noted that depression, whether in the clinical assessment (CAMDEX) $\left(F_{1,117}=2.5\right.$, $\left.p=0.111, \eta_{p}^{2}=0.02\right)$ or when measured by the corresponding NPI subscale $\left(F_{1,117}=2.0, p=0.159\right.$, $\left.\eta^{2}=0.01\right)$, was not associated with discrepancies in perceived QoL-p. In both patients and caregivers, depression in the patient had a negative effect on ratings of QoL-p, both at baseline and at 36 months (see regression analysis).

\section{Anosognosia, cognition, and patient and caregiver ratings of $Q o L-p$}

The presence of anosognosia was associated with greater cognitive impairment after 12 months, as measured by both the CAMCOG $\left(F_{1,117}=10.7, p=0.001\right.$, $\left.\eta_{p}^{2}=0.08\right)$ and the MMSE $\left(F_{1,117}=5.0, p=0.026\right.$, $\left.\eta_{p}^{2}=0.04\right)$.

In terms of QoL-p, patients with anosognosia gave higher ratings of their quality of life $\left(F_{1,117}=7.0\right.$, $\left.p=0.009, \eta_{p}^{2}=0.05\right)$, especially after 12 months. However, cognitive status, whether measured by the CAMCOG $\left(F_{1,117}=0.0, p=0.928, \eta^{2}{ }_{p}=0.00\right)$ or the $\operatorname{MMSE}\left(F_{1,117}=0.1, p=0.675, \eta^{2}{ }_{p}=0.00\right)$, had no overall effect on their QoL-p ratings. Only for the MMSE was there a significant effect at baseline, where higher ratings of QoL-p were given by patients with better cognitive function $(z=2.4, p=0.015, d=0.036)$.

For caregivers, anosognosia had no significant effect on their ratings of QoL-p $\left(F_{1,117}=0.7, p=0.373\right.$, $\left.\eta_{p}^{2}=0.00\right)$. Although cognitive status, as measured by both the CAMCOG $\left(F_{1,117}=0.4, p=0.493\right.$, $\left.\eta^{2} p=0.00\right)$ and the MMSE $\left(F_{1,117}=2.4, p=0.118\right.$, $\left.\eta_{p}^{2}=0.02\right)$, had no overall effect on their ratings of QoL-p, there was a significant effect for CAMCOG scores at 36 months $\left(t_{117}=2.3, p=0.019, d=0.047\right)$, with lower ratings being given to patients with greater cognitive impairment.

The data regarding the relationship between anosognosia, cognition, and patient and caregiver ratings of QoL-p can be consulted in Supplementary Table 1.

\section{DISCUSSION}

Mention should first be made of the high number of cases lost to follow-up, this being a common feature of longitudinal studies of patients with AD. The percentage of lost cases $(64.7 \%)$ is similar to that reported in other studies conducted over three years $(69.1 \%)$ [14] or two years $(64.3 \%)$ [12], $(60.8 \%)$ [13]. As in these studies, the patients lost to follow-up tended to be older and more impaired.

\section{Patient and caregiver perceptions of the patient's quality of life}

The first objective of this study was to determine changes over time in patient and caregiver ratings of QoL-p. The results show that patients' ratings remained generally stable over the follow-up period, despite their deteriorating status. By contrast, caregiver ratings showed a clear decline as the patient became more impaired.

The second objective was to identify the factors associated with perceived QoL-p. The results for patients show that depression was associated with lower ratings of QoL-p, a finding that is consistent with previous cross-sectional $[17,28]$ and longitudinal studies [8]. Conversely, greater anosognosia was related to better perceived QoL-p [29-31]. Male gender and a higher level of education were also associated with higher ratings of QoL-p.

The perception of caregivers was heavily influenced by the patient's functional status, both at baseline and during follow-up, while over time neuropsychiatric symptoms (depression, apathy, and agitation) came to have a greater effect. Functional deficits [11, 14] and neuropsychiatric symptoms $[10,11]$ are the factors most often cited in previous research as having a negative impact on caregiver ratings of QoL-p. In the present results, it is also important to note the minimal influence of cognitive status, which was only significant in relation to caregiver ratings at 36 months. Caregiver burden remained a significant factor at all times, although its negative effect was greater at baseline, as was the case for poorer mental health.

These results highlight the considerable subjectivity associated with the concept of quality of life. In the early stages of dementia the positive ratings of patients can be understood in terms of psychological mechanisms, the disability paradox (adaptation to change) [32], or processes of "self-maintaining" and "self-adjusting" [33]. The more negative ratings of caregivers can be explained by the impact of the diagnosis and the functional and behavioral changes in the patient, which would produce greater burden.

However, these concepts are not sufficient to explain the results for perceived QoL-p in the advanced stages of $\mathrm{AD}$. Here, greater anosognosia in patients, related to increased neurological deterioration, would lead them to have an overly positive view of their quality of life, one that differs considerably from their 
actual status. At this point, the more negative rating of caregivers is consistent with the patient's deteriorating state. Note, however, that the smaller effect of burden on caregiver ratings of QoL-p at 36 months would be related to their increased adaptation to the situation [34].

\section{Discrepancies between patient and caregiver ratings of the patient's quality of life}

The third objective of this study was to identify any discrepancies between patient and caregiver ratings of QoL-p. Caregiver ratings were always more negative than those of patients, a finding corroborated by various studies $[10,11,13,14]$. However, the present study makes a novel contribution by analyzing the specific factors that might explain the observed discrepancies.

The first discrepancy is associated with the presence of neuropsychiatric symptoms, especially agitation [35] and apathy [35, 36]. Caregiver ratings were lower in the presence of these symptoms, most likely as a result of the distress they produce. By contrast, there was greater agreement in the presence of depression in the patient, which had a negative effect on the ratings of both patients $[8,11,35-38]$ and caregivers [14, 35, 36]. The impact of depression in this regard has been widely reported.

The second discrepancy relates to anosognosia and its effect on perceived QoL-p. This aspect has not been widely investigated, although two cross-sectional studies addressing this important topic have recently been published [31, 39]. Greater anosognosia led patients to have a more positive view of their quality of life, despite their general deterioration. This lack of awareness regarding their actual status suggests that their perceptions correspond to a mental image of themselves prior to their deterioration [40].

The third discrepancy concerns the patient's functional status. Although functional deficits had a negative effect on patients themselves, especially during the first year of follow-up when they were more aware of their condition, the greatest effect was on caregivers, in whom the patient's functional impairment produced a negative view of QoL-p throughout the follow-up period [5, 14, 35, 36, 41].

Finally, the fourth discrepancy relates to caregiver burden. Higher levels of burden were associated with a greater discrepancy between patient and caregiver ratings of QoL-p [37]. The main correlates of caregiver burden were the severity of neuropsychiatric symptoms, poorer functional status, and lower ratings of the patient's quality of life [42]. A high degree of anosog- nosia has previously been associated with greater burden [43]. Very high levels of caregiver burden could lead to overly negative views of the patient's status, especially with regard to neuropsychiatric symptoms and the patient's quality of life [34, 44, 45].

In contrast to these discrepancies, depression in the patient had a negative effect on the QoL-p ratings of both patients and caregivers. These results are consistent with previous studies of caregivers [14] and patients $[8,17,28]$.

\section{Anosognosia, cognition, and patient and caregiver ratings of $Q o L-p$}

A specific aspect to consider is the relationship between anosognosia, cognitive status, and perceived quality of life. The present findings confirm the results of a previous cross-sectional study [30], with anosognosia being associated with greater impairment among patients with moderate dementia. More specifically, anosognosia was associated with better perceived QoL-p in patients with moderate dementia, whereas cognition was only associated with higher ratings of perceived QoL-p among those with mild dementia. In conclusion, among patients: a) anosognosia was associated with cognitive impairment; b) anosognosia was associated with better perceived QoL-p in moderate dementia, whereas cognitive status did not influence the ratings of these patients; and c) anosognosia and cognition act as independent variables in relation to perceived quality of life. Among caregivers, anosognosia did not influence their perceptions of QoL-p, and cognitive impairment only had a negative effect on QoL-p ratings at 36 months.

\section{Limitations of study}

One important limitation, associated with longitudinal studies of patients with AD, is the loss to follow-up of older patients and those with more advanced dementia, although in many of these patients (with a MMSE score $<10$ ), the quality of life scale would not have been applicable. This loss of patients may have led to a slight increase in QoL-p scores, both among caregivers and patients themselves.

Although, in the global analysis, patients' scores on the QoL-AD remained stable over time there were notable differences between individuals. However, for reasons of space the present article has not discussed the factors associated with the sub-groups of patients whose scores showed a decline, stability, or improvement during follow-up. This aspect will be analyzed in 
subsequent research. A further limitation is that while the clinical assessment of anosognosia provides important information, the data are limited by the lack of a specific instrument for this purpose. This issue should be resolved in future longitudinal studies. Finally, it should be noted that certain variables (e.g., hours per day spent on caring, resources available, time since taking up caregiving role, or comorbidities) could not be controlled due to a lack of data.

\section{CONCLUSIONS}

Patients' ratings of their own quality of life remained stable over the follow-up period. The factor that had the most negative and consistent effect on their ratings was depression. Caregiver ratings of the patient's quality of life showed a significant decline over time, such that the discrepancies between patient and caregiver ratings increased over follow-up. These discrepancies were related to greater anosognosia, agitation, apathy, and functional impairment in the patient, and to burden and worse mental health in the caregiver. All these factors should be taken into account when quality-oflife measures are used to evaluate the effectiveness of therapeutic interventions for patients with AD.

There is obviously a need to find ways of improving caregivers' perceptions of the patient's status so as to help reduce the burden they experience, since stress in the caregiver can impact negatively on the patient. Psychoeducational and therapeutic interventions aimed at family caregivers may be one way of enabling them to learn more about dementia, to improve their relationship with the patient, and to help them cope with the stress that is an inherent part of caring for someone with AD [46-49].

The most important contributions of the present longitudinal study are as follows: 1) It identifies the variables related to the greatest discrepancies in patient and caregiver ratings of QoL-p; 2) It confirms that depression in the patient does not produce discrepancies, since the ratings of both patients and caregivers were negatively affected by this variable; and 3) Anosognosia and cognition are shown to be independent variables in relation to patients' perceptions of their quality of life.

\section{ACKNOWLEDGMENTS}

The research was conducted in the Memory and Dementia Assessment Unit of the Santa Caterina Hospital (Salt, Spain) and formed part of the SIDEA research project (Seguimiento Integral de la Enfermedad de Alzheimer/Comprehensive Follow-Up of Alzheimer's Disease, 2003-12).

Authors' disclosures available online (http://www.jalz.com/disclosures/view.php?id=1966).

\section{SUPPLEMENTARY MATERIAL}

The supplementary table is available in the electronic version of this article: http://dx.doi.org/10.3233/ JAD-131286.

\section{REFERENCES}

[1] Kurz A, Schulz M, Reed P, Wortmann M, Rodrigo J, von Lützau Hohlbein H, Grosssberg G (2008) Personal perspectives of persons with Alzheimer's disease and their carers: A global survey. Alzheimers Dement 4, 345-352.

[2] Kivipelto M, Solomon A, Ahtiluoto S, Ngandu T, Lehtisalo J, Antikainen R, Bäckman L, Hänninen T, Jula A, Laatikainen T, Lindström J, Mangialasche F, Nissinen A, Paajanen T, Pajala S, Peltonen M, Rauramaa R, Stigsdotter-Neely A, Strandberg T, Tuomilehto J, Soininen H (2013) The Finnish Geriatric Intervention Study to Prevent Cognitive Impairment and Disability (FINGER): Study design and progress. Alzheimers Dement 9, 657-665.

[3] Lyketsos CG, Miller DS; Neuropsychiatric Syndromes Professional Interest Area of the International Society to Advance Alzheimer's Research and Treatment (2012) Addressing the Alzheimer's disease crisis through better understanding, treatment, and eventual prevention of associated neuropsychiatric syndromes. Alzheimers Dement 8, 60-64.

[4] Katona C, Livingston G, Cooper C, Ames D, Brodaty H, Chiu E (2007) International Psychogeriatric Association consensus statement on defining and measuring treatment benefits in dementia. Int Psychogeriatr 19, 345-354.

[5] Cooper C, Mukadam N, Katona C, Lyketsos CG, Ames D, Rabins P, Engedal K, de Mendonça Lima C, Blazer D, Teri L, Brodaty H, Livingston G, World Federation of Biological Psychiatry - Old Age, Taskforce (2012) Systematic review of the effectiveness of non-pharmacological interventions to improve quality of life of people with dementia. Int Psychogeriatr 24, 856-870.

[6] Vogel A, Mortensen EL, Hasselbalch SG, Andersen BB, Waldemar G (2006) Patient versus informant reported quality of life in the earliest phases of Alzheimer's disease. Int $J$ Geriatr Psychiatry 21, 1132-1138.

[7] Ready RE, Ott BR, Grace J (2006) Insight and cognitive impairment: Effects on quality-of-life reports from mild cognitive impairment and Alzheimer's disease patients. Am J Alzheimers Dis Other Demen 21, 242-248.

[8] Selwood A, Thorgrimsen L, Orrell M (2005) Quality of life in dementia-a one-year follow-up study. Int J Geriatr Psychiatry 20, 232-237.

[9] Karlawish JH, Zbrozek A, Kinosian B, Gregory A, Ferguson A, Low DV, Glick HA (2008) Caregivers' assessments of preference-based quality of life in Alzheimer's disease. Alzheimers Dement 4, 203-211.

[10] Jönsson L, Andreasen N, Kilander L, Soininen H, Waldemar G, Nygaard H, Winblad B, Jönhagen ME, Hallikainen M, Wimo A (2006) Patient and proxy-reported utility in 
Alzheimer disease using the EuroQoL. Alzheimer Dis Assoc Disord 20, 49-55.

[11] Tatsumi H, Nakaaki S, Torii K, Shinagawa Y, Watanabe N, Murata Y, Sato J, Mimura M, Furukawa TA (2009) Neuropsychiatric symptoms predict change in quality of life of Alzheimer disease patients: A two-year follow-up study. Psychiatry Clin Neurosci 63, 374-384.

[12] Missotten P, Ylieff M, Di Notte D, Paquay L, De Lepeleire J, Buntinx F, Fontaine O (2007) Quality of life in dementia: A 2-year follow-up study. Int J Geriatr Psychiatry 22, 12011207.

[13] Lyketsos CG, Gonzales-Salvador T, Chin JJ, Baker A, Black B, Rabins P (2003) A follow-up study of change in quality of life among persons with dementia residing in a long-term care facility. Int J Geriatr Psychiatry 18, 275-281.

[14] Vogel A, Bhattacharya S, Waldorff FB, Waldemar G (2012) Proxy-rated quality of life in Alzheimer's disease: A threeyear longitudinal study. Int Psychogeriatr 24, 82-89.

[15] American Psychiatric, Association (2001) Manual diagnóstico y estadístico de los trastornos mentales, 4th ed., texto revisado (DSM-IV-TR), Masson, Barcelona.

[16] McKhann G, Drachman D, Folstein M, Katzman R, Price D, Stadlan EM (1984) Clinical diagnosis of Alzheimer's disease: Report of the NINCDS-ADRDA Work Group under the auspices of Department of Health and Human Services task force on Alzheimer's disease. Neurology 34, 939-944.

[17] Conde-Sala JL, Garre-Olmo J, Turró-Garriga O, López-Pousa S, Vilalta-Franch J (2009) Factors related to perceived quality of life in patients with Alzheimer's disease: The patient's perception compared with that of caregivers. Int J Geriatr Psychiatry 24, 585-594.

[18] Logsdon RG, Gibbons LE, McCurry SM, Teri L (2002) Assessing quality of life in older adults with cognitive impairment. Psychosom Med 64, 510-519.

[19] Roth M, Tym E, Mountjoy CQ, Huppert FA, Hendrie H, Verma S, Goddard R (1986) CAMDEX. A standardised instrument for the diagnosis of mental disorder in the elderly with special reference to the early detection of dementia. $\mathrm{Br}$ J Psychiatry 149, 698-709.

[20] Folstein MF, Folstein SE, McHugh PR (1975) 'Mini Mental State'. A practical method for grading the cognitive state of patients for the clinician. J Psychiatr Res 12, 189-198.

[21] Gélinas I, Gauthier L, McIntyre M, Gauthier S (1999) Development of a functional measure for persons with Alzheimer's disease: The Disability Assessment for Dementia. Am J Occup Ther 53, 471-481.

[22] Cummings JL, Mega M, Gray K, Rosemberg-Thompson S, Carusi DA, Gornbein J (1994) The neuropsychiatric inventory. Comprehensive assessment of psychopathology in dementia. Neurology 44, 2308-2314.

[23] Ware JE Jr, Kosinski M, Keller SD (1996) A 12-Item Short-Form Health Survey: Construction of scales and preliminary tests of reliability and validity. Med Care 34, 220-233.

[24] Zarit SH, Todd PA, Zarit JM (1986) Subjective burden of husbands and wives as caregiver: A longitudinal study. Gerontologist 26, 260-266.

[25] Guilford JP, Fruchter B (1973) Fundamental Statistics in Psychology and Education, 5th ed, McGraw-Hill, New York.

[26] Cohen J (1973) Eta-squared and partial eta-squared in fixed factor ANOVA designs. Educ Psychol Meas 33, 107-112.

[27] Cohen J (1988) Statistical Power Analysis for the Behavioral Sciences, 2nd ed. Erlbaum, Hillsdale, NJ.

[28] Naglie G, Hogan DB, Krahn M, Beattie BL, Black SE, Macknight C, Freedman M, Patterson C, Borrie M, Bergman H,
Byszewski A, Streiner D, Irvine J, Ritvo P, Comrie J, Kowgier M, Tomlinson G (2011) Predictors of patient self-ratings of quality of life in alzheimer disease: Cross-sectional results from the Canadian Alzheimer's Disease Quality of Life Study. Am J Geriatr Psychiatry 19, 881-890.

[29] Berwig M, Leicht H, Gertz HJ (2009) Critical evaluation of self-rated quality of life in mild cognitive impairment and Alzheimer's disease-further evidence for the impact of anosognosia and global cognitive impairment. J Nutr Health Aging 13, 226-230.

[30] Hurt CS, Banerjee S, Tunnard C, Whitehead DL, Tsolaki M, Mecocci P, Kloszewska I, Soininen H, Vellas B, Lovestone S (2010) Insight, cognition and quality of life in Alzheimer's disease. J Neurol Neurosurg Psychiatry 81, 331-336.

[31] Conde-Sala JL, Reñé-Ramírez R, Turró-Garriga O, GascónBayarri J, Campdelacreu-Fumadó J, Juncadella-Puig M, Rico-Pons I, Garre-Olmo J (2013) Severity of dementia, anosognosia and depression in relation to the quality of life of patients with Alzheimer's disease: Discrepancies between patients and caregivers. Am J Geriatr Psychiatry, doi: 10.1016/j.jagp.2012.07.001.

[32] Ettema TP, Dröes RM, de Lange J, Ooms ME, Mellenbergh GJ, Ribbe MW (2005) The concept of quality of life in dementia in the different stages of the disease. Int Psychogeriatr 17, 353-370.

[33] Clare L (2003) Managing threats to self: Awareness in early stage Alzheimer's disease. Soc Sci Med 57, 1017-1029.

[34] Conde-Sala JL, Reñé-Ramírez R, Turró-Garriga O, GascónBayarri J, Juncadella-Puig M, Moreno-Cordón L, Viñas-Díez V, Vilalta-Franch J, Garre-Olmo J (2013) Factors associated with the variability in caregiver assessments of the capacities of patients with Alzheimer disease. J Geriatr Psychiatry Neurol 26, 86-94.

[35] Karttunen K, Karppi P, Hiltunen A, Vanhanen M, Välimäki T, Martikainen J, Valtonen H, Sivenius J, Soininen H, Hartikainen S, Suhonen J, Pirttilä T, study ALSOVA, group (2011) Neuropsychiatric symptoms and quality of life in patients with very mild and mild Alzheimer's disease. Int $J$ Geriatr Psychiatry 26, 473-482.

[36] Hoe J, Katona C, Orrell M, Livingston G (2007) Quality of life in dementia: Care recipient and caregiver perceptions of quality of life in dementia: The LASER-AD study. Int J Geriatr Psychiatry 22, 1031-1036.

[37] Gómez-Gallego M, Gómez-Amor J, Gómez-García J (2012) Determinants of quality of life in Alzheimer's disease: Perspective of patients, informal caregivers, and professional caregivers. Int Psychogeriatr 24, 1805-1815.

[38] Hoe J, Hancock G, Livingston G, Woods B, Challis D, Orrell M (2009) Changes in the quality of life of people with dementia living in care homes. Alzheimer Dis Assoc Disord 23, 285-290.

[39] Conde-Sala JL, Reñé-Ramírez R, Turró-Garriga O, GascónBayarri J, Juncadella-Puig M, Moreno-Cordón L, Viñas-Díez L, Garre-Olmo J (2013) Clinical differences in patients with Alzheimer's disease according to the presence or absence of anosognosia: Implications for perceived quality of life. $J$ Alzheimers Dis 33, 1105-1116.

[40] Agnew SK, Morris RG (1998) The heterogeneity of anosognosia for memory impairment in Alzheimer's disease: A review of the literature and a proposed model. Aging Ment Health 2, 7-19.

[41] Karlawish JH, Zbrozek A, Kinosian B, Gregory A, Ferguson A, Glick HA (2008) Preference-based quality of life in patients with Alzheimer's disease. Alzheimers Dement 4, 193202. 
[42] Mohamed S, Rosenheck R, Lyketsos CG, Schneider LS (2010) Caregiver burden in Alzheimer disease: Crosssectional and longitudinal patient correlates. Am J Geriatr Psychiatry 18, 917-927.

[43] Turró-Garriga O, Garre-Olmo J, Vilalta-Franch J, Conde-Sala J, de Gracia-Blanco M, López-Pousa S (2013) Burden associated with the presence of anosognosia in Alzheimer's disease. Int J Geriatr Psychiatry 28, 291-297.

[44] Karlawish JH, Casarett D, Klocinski J, Clark CM (2001) The relationship between caregivers' global ratings of Alzheimer's disease patients' quality of life, disease severity, and the caregiving experience. $J$ Am Geriatr Soc 49 1066-1070.

[45] Schulz R, Cook TB, Beach SR, Lingler JH, Martire LM, Monin JK, Czaja SJ (2013) Magnitude and causes of bias among family caregivers rating Alzheimer disease patients. Am J Geriatr Psychiatry 21, 14-25.

[46] Pinquart M, Sörensen S (2006) Helping caregivers of persons with dementia: Which interventions work and how large are their effects? Int Psychogeriatr 18, 577-595.
[47] Gallagher-Thompson D, Coon DW, Solano N, Ambler C, Rabinowitz Y, Thompson LW (2003) Change in indices of distress among Latino and Anglo female caregivers of elderly relatives with dementia: Site-specific results from the REACH national collaborative study. Gerontologist 43, 580-591.

[48] Gitlin LN, Hauck WW, Dennis MP, Winter L (2005) Maintenance of effects of the home environmental skillbuilding program for family caregivers and individuals with Alzheimer's disease and related disorders. J Gerontol A Biol Sci Med Sci 60, 368-374.

[49] Belle SH, Burgio L, Burns R, Coon D, Czaja SJ, GallagherThompson D, Gitlin LN, Klinger J, Koepke KM, Lee CC, Martindale-Adams J, Nichols L, Schulz R, Stahl S, Stevens A, Winter L, Zhang S, Resources for Enhancing Alzheimer's Caregiver Health (REACH) II, Investigators (2006) Enhancing the quality of life of dementia caregivers from different ethnic or racial groups: A randomized, controlled trial. Ann Intern Med 145, 727-738. 